\title{
¿Por qué es importante detectar la gastritis atrófica y la metaplasia intestinal gástrica? ¿Cuál es la forma adecuada de hacerlo?
}

\author{
Why is it important to detect atrophic gastritis and gastric intestinal metaplasia? What \\ is the proper way to do it?
}

Muhannad Sarem ${ }^{1,2}$, Rodolfo Corti ${ }^{3,4}$

${ }^{1}$ Unidad de Endoscopia Digestiva, Hospital Dr. Diego Thompson. Buenos Aires, Argentina.

2 Departamento de Ciencias Morfológicas, Facultad de Medicina, Instituto Universitario de Ciencias de la Salud, Fundación Héctor A. Barceló. La Rioja, Argentina.

${ }^{3}$ Unidad de Esofago y Estomago, Hospital de Gastroenterología Bonorino Udaondo. Buenos Aires, Argentina.

${ }^{4}$ Unidad Académica Bonorino Udaondo Facultad de Medicina, Universidad de Buenos Aires. Buenos Aires, Argentina.

Recibido: 29/06/2020 - Aprobado: 17/08/2020

ORCID: Muhannad Sarem: https://orcid.org/0000-0001-8521-9076, Rodolfo E Corti: https://orcid.org/0000-0002-6573-3075

\section{RESUMEN}

La metaplasia intestinal gástrica y la gastritis atrófica son condiciones precancerosas conocidas (CPCs) del estómago, lo que significa que los pacientes con CPCs están en riesgo de desarrollar cáncer gástrico y, por lo tanto, el diagnóstico y la categorización de riesgo para estos pacientes es un tema relevante. El objetivo de esta revisión es proporcionar una actualización sobre el problema, el diagnóstico y el manejo de las CPCs con énfasis en el papel de la detección endoscópica adecuada.

Palabras clave: Gastritis atrófica; Metaplasia; Condiciones precancerosas (fuente: DeCS BIREME).

\section{ABSTRACT}

Gastric intestinal metaplasia and atrophic gastritis are a known precancerous condition (PCC) of the stomach, meaning that patients with PCC are at risk for gastric cancer and so, diagnosis and risk categorization for these patients is relevant. The aim of this review is to provide an update regarding the problem, diagnosis, and management of PCCs with an emphasis on the role of appropriate endoscopic detection.

Keywords: Gastritis, atrophic; Metaplasia; Precancerous condition (source: MeSH NLM).

\section{INTRODUCCIÓN}

El cáncer gástrico (CG) es el quinto cáncer más común en todo el mundo y la tercera causa de muerte relacionada con el cáncer. Las áreas de alta incidencia son Asia Oriental, Europa del Este, América Central y del Sur; la mayoría de los casos se diagnostican en una etapa tardía, lo que significa que la mayoría de los pacientes con diagnóstico de CG muere de la enfermedad ${ }^{(1,2)}$. Sin embargo, la detección y vigilancia de las personas en riesgo puede disminuir la mortalidad por este cáncer ${ }^{(3-5)}$. Por ejemplo en el año 1983, el gobierno japonés emitió una ley que exige establecer un sistema de detección del CG. Como resultado de esta política, aproximadamente el 60\% del CG se detecta en su estadio temprano ${ }^{(6)}$. Es ampliamente sabido que el pronóstico del cáncer gástrico temprano (CGT) es excelente con una tasa de supervivencia a 5 años superior al $90 \%{ }^{(7,8)}$, mientras que, a modo comparativo, la tasa de supervivencia a 5 años de pacientes con CG proximal es tan solo del 10 al $15 \%{ }^{\left({ }^{9}\right)}$. Por lo tanto, probablemente la mejor manera de cambiar el pronóstico del CG es detectandolo en su etapa de evolución temprana y, más aún, detectando a tiempo las condiciones y lesiones precancerosas.

En este artículo, trataremos de remarcar la relevancia de la detección de las condiciones precancerosas (CPCs) ya que son las que conllevan al desarrollo de lesiones precancerosas (LPCs) y del CGT, ambas lesiones pueden ser tratadas endoscópicamente.

\section{Inflamación crónica de la mucosa gástrica}

Tradicionalmente el CG se subdivide, según la clasificación de Lauren, en dos tipos principales, el CG de tipo intestinal (CGI) y el CG de de tipo difuso $(C G D)^{(10)}$. El CGI representa el resultado final de la ya conocida cascada de Correa, una secuencia de progresión dada por inflamación, atrofia, metaplasia, displasia y carcinoma ${ }^{(11)}$. Es importante tener en cuenta que la atrofia de la mucosa es el resultado final de una enfermedad inflamatoria crónica de la mucosa gástrica independiente de la etiología. Tanto la infección por el Helicobacter pylori como la gastritis autoinmune, son las principales causas de esta inflamación crónica, 
es decir gastritis crónica (GC) ${ }^{(12-14)}$. Aunque también existen otras etiologías que conducen a la GC y que no serán tratadas en este texto ${ }^{(15)}$, creemos que vale la pena mencionar que el reflujo duodeno-gástrico, una condición relativamente frecuente, es otra causa de daño a nivel de la mucosa gástrica que puede contribuir al desarrollo de GC y avanzar luego a metaplasia, sería un proceso casi análogo al reflujo gastroesofágico en el esófago de Barrett ${ }^{(16)}$.

\section{Condiciones y lesiones precancerosas}

La GC puede conducir a la gastritis atrófica (GA) que se refiere a la atrofia y pérdida de las glándulas gástricas en un determinado sector gástrico. En cambio la metaplasia intestinal gástrica (MIG), se define como el reemplazo de células columnares gástricas por células de morfología intestinal caracterizada por la presencia de células caliciformes, células de Paneth, y enterocitos ${ }^{(17)}$. La MIG puede subclasificarse en MIG completa e incompleta. La MIG completa o de tipo intestinal se asemeja al epitelio del intestino delgado, mientras que la MIG incompleta o de tipo colónica se parece más al epitelio del colon; la distinción entre MIG completa e incompleta es clínicamente importante ya que parece que la MIG incompleta tiene mayor riesgo de progresión a cáncer ${ }^{(18,19)}$.

\section{Gastritis atrófica:}

Como hemos mencionado arriba, el desarrollo de GA comienza en una cascada de cambios en la mucosa y que puede progresar a MIG y finalmente a CG. Como es de esperar, la tasa de incidencia de progresión de GA a CG varía debido a la heterogeneidad de los estudios y la ubicación geográfica estudiada oscilando entre 1,0 y 5,2 por 1000 personas/año ${ }^{(20,21)}$. El grado de atrofia es un factor relevante ya que la presencia de GA leve a moderada se asocia con un aumento de 6.4 veces de la incidencia de CG mientras que la GA severa se asocia a un aumento casi el doble (de 11,8 veces) ${ }^{(22)}$. Del mismo modo, la extensión de la GA también es importante ya que los pacientes con GA extensa tienen mayor riesgo de desarrollar $\mathrm{CG}^{(23)}$.

\section{Metaplasia intestinal gástrica:}

Una vez establecida, se ha demostrado que el grado de la MIG está relacionado con el riesgo de progresión a cáncer. La MIG extensa con MIG en el cuerpo, la MIG incompleta y la MIG ubicada a lo largo de la "calle gástrica" aumenta el riesgo de progresión hacia el CG ${ }^{(19,24,25)}$.

De modo similar a los estudios de incidencia de la GA, las tasas de incidencia de la MIG calculadas en una revisión sistemática varían entre 1,26 y 4,10 por 1000 personas/año ${ }^{(21)}$. En la Argentina, Corti et al. publicaron 2 trabajos en relación a la prevalencia de MIG en biopsias de pacientes con síntomas de tubo digestivo superior con más de 2000 pacientes evaluados en cada estudio. El primero en el año 2001 la prevalencia de MIG fue del 7,9\% y el segundo en el año 2012 fue del $11 \%{ }^{(26,27)}$. Estos datos son coincidentes con el concepto de que Argentina es un país de riesgo intermedio de cáncer gástrico (6-7/100 000 personas/año). En una investigación reciente se obtuvo un valor casi idéntico aunque los pacientes asiáticos que viven en Argentina tendrían un mayor riesgo ${ }^{(28)}$.

Aun así, se demostró que entre hermanos con antecedentes familiares de cualquier cambio precanceroso, existe un aumento en el riesgo de desarrollo de CG de 2,5 en comparación con los hermanos de personas con "mucosa normal o de menor cambios" (29), lo que refleja que la disponibilidad de datos sobre CPCs de los hermanos podrían ser útiles para evaluar el riesgo de un paciente de progresar a CG.

En un estudio de cohorte a nivel nacional en Los Países Bajos, se demostró que la incidencia anual del CG es del $0,1 \%$ para pacientes con GA, 0,25\% para MIG, 0,6\% para displasia leve a moderada y $6 \%$ para displasia severa dentro de los 5 años posteriores al diagnóstico ${ }^{(30)}$. Estos datos fueron avalados por otro estudio sueco donde se halló que la incidencia de CG es mayor en pacientes con GA y MIG; las tasas de incidencia observadas en este estudio predicen que dentro de los 20 años posteriores a la endoscopía digestiva alta (EDA), el CG se desarrollará en aproximadamente 1 en 50 paciente con GA, 1 en 39 pacientes con MIG y 1 en 19 pacientes con displasia ${ }^{(31)}$. Otros estudios y metaanálisis demostraron resultados similares ${ }^{(32-34)}$.

Por lo tanto, la GA y la MI se consideran condiciones precancerosas (CPCs) porque constituyen el trasfondo en el que pueden ocurrir displasia y adenocarcinoma ${ }^{(35)}$.

\section{Displasia intraepitelial:}

La displasia intraepitelial gástrica (DIG), se define como un epitelio neoplásico sin evidencia de invasión tisular (de la lámina propia); representa la penúltima etapa de la secuencia de carcinogénesis gástrica por lo que se considera una lesión precancerosa (LPC) ${ }^{(35)}$. La DIG se clasifica en displasia de bajo grado (DBG) y displasia de alto grado (DAG). La mayoría de los pacientes que albergan lesiones clasificadas como DAG tienen alto riesgo de un rápido progreso a CG o de tener concomitantemente un carcinoma invasivo dentro de esa lesión o en forma sincrónica ${ }^{(36)}$. Es notable que el $25 \%$ de las lesiones con DBG pueden estar sub-categorizadas (de forma similar a la que ocurre en el esófago de Barrett ${ }^{(37)}$ y el $7 \%$ de estos ya tiene un carcinoma presente en la LPC (38). En un 
estudio realizado en nuestro país, se incluyeron 39 pacientes con MIG comprobada histológicamente, y se encontró que el 31\% de ellos presentaron displasia en al menos una de cuatro regiones anatómicas del estómago (curvatura mayor y menor del cuerpo, ángulo y antro). La presencia de displasia se asoció con el origen asiático, al menos una lesión metaplásica deprimida, más de una región gástrica afectada y compromiso de la mucosa oxíntica (ambas curvaturas del cuerpo) por lo que los autores recomiendan especial atención en el examen endoscópico, así como la toma de múltiples biopsias dirigidas, cuando estas características endoscópicas y clínicas, que fueron tomados en cuenta en este estudio, estén presentes ${ }^{(39)}$.

\section{OLGA y OLGIM}

El sistema OLGA (Operative Link on Gastritis Assessment) es una escala para evaluar y determinar la extensión de la atrofia según un grado de riesgo de CG que aumenta progresivamente, desde el estadio más bajo (estadio 0) hasta el más alto (estadio IV) ${ }^{(40,41)}$. Dicho sistema solo puede ser aplicado cuando se dispone de un conjunto de por lo menos cinco biopsias tomadas según el sistema de Sydney. Varios estudios de seguimiento han demostrado que el sistema OLGA permite identificar los pacientes con mayor riesgo de desarrollar CG si están categorizados dentro de los estadios III o IV ${ }^{(42)}$. Como efecto lógico, se debe ofrecer un seguimiento endoscópico a aquellos pacientes que están en estas subcategorías ${ }^{(35,42)}$.

Luego, un grupo de expertos de Los Países Bajos propuso una modificación del sistema OLGA, el sistema OLGIM (Operative Link on Gastritis Assessment based on Intestinal Metaplasia) que utiliza los mismos conceptos, pero reemplaza la escala de atrofia por la determinación de la MIG, ya que ésta es más fácil de reconocer por los patólogos, es más fácil de evaluar y tiene mejor acuerdo interobservador ${ }^{(43,44)}$. Al igual que el sistema OLGA, las etapas OLGIM III O IV pueden identificar a los pacientes con mayor riesgo de desarrollar CG.

\section{Condiciones precancesosas y cáncer gástrico difuso}

Tal como hemos mencionado arriba, la mayoría de los cánceres gástricos se subdividen principalmente en CGI y CGD. Aunque existe una considerable evidencia de que la MIG progresa a displasia y luego a CGI, estudios recientes revelan que las CPCs de la cascada de Correa tendrían alguna relación con la carcinogénesis del CGD.

En un estudio japonés prospectivo, se sugirió una asociación significativa entre MIG y el desarrollo del
CGD ${ }^{(45)}$. Un estudio más reciente en Corea del Sur mostró que los antecedentes familiares de CG, la infección por el H. pylori y los estadíos OLGA / OLGIM III / IV fueron factores de riesgo independientes tanto para el CGI como para el CGD ${ }^{(46)}$. Otro estudio del mismo país asiático publicado recientemente reveló que entre los pacientes con CGD, el 52,5\% de los ellos tenía GA y el $18,4 \%$ tenía GA grave. Con respecto a la MIG, el $42,1 \%$ de los pacientes tenía MIG y el $17,1 \%$ tenía MI severa. El CGD combinado con GA o MIG severa mostró un tamaño tumoral mayor y una tasa de invasión submucosa más alta que aquella sin GA o MIG severa ${ }^{(47)}$. Por lo tanto, las CPCs probablemente jueguen un doble papel en la carcinogénesis gástrica dependiente del contexto.

En nuestro país, Kujaruk et al. examinaron la mucosa adyacente al CG en 76 estómagos resecados. Se encontró que la MIG estaba presente en el $83 \%$ de los CGI, en el 59\% de los CGD y en el 100\% de los mixtos e indeterminados. Con OLGA, mostraron atrofia avanzada el $41 \%$ de los CGI, 12\% de los CGD, $25 \%$ de los mixtos y $45 \%$ de los indeterminados ${ }^{(48)}$. Si bien la muestra fue pequeña, está claro que las CPCs están presentes tanto en el CGI como en el CGD y los mixtos e indeterminados y tal vez fueron un marcador fiel que no fue aprovechado como tal mediante su detección temprana en una "hipotética" EDA previa al diagnóstico del CG.

\section{Detección endoscópica de las condiciones precancerosas}

Con los endoscopios convencionales de luz blanca (E-CLB), la correlación histológica con la apariencia endoscópica de mucosa atrófica y/o metaplásica no es alta ${ }^{(49,50)}$, aunque la tinción con colorantes vitales, o cromoendoscopia tradicional, (CE-tradicional) mejora notoriamente su reconocimiento ${ }^{(51,52)}$. En un estudio de 376 pacientes, Calle Astudillo et al. compararon la utilidad de biopsias tomadas al azar con las dirigidas por tinción de azul de metileno para el diagnóstico de MIG. La especificidad fue del $91 \%$ y la sensibilidad del $46 \%$ para el diagnóstico endoscópico y del 67\% y 95\% para el diagnóstico con azul de metileno lo que demuestra una diferencia significativa a favor de la tinción ${ }^{(53)}$. En otro estudio de 126 participantes, la precisión diagnóstica general de la CE-tradicional con ácido acético fue de $89 \%$ con una especificidad y sensibilidad del $94,4 \%$ y $77,6 \%$ respectivamente. La proporción de la MIG extensa, aumentó de $0,9 \%$ a 18,1\% cuando se usó ácido acético en lugar de E-CLB ${ }^{(54)}$. El uso de bicromoendoscopia con una mezcla de ácido acético e índigo carmín demostró resultados similares ${ }^{(55)}$. Sin embargo, la CE-tradicional no suele utilizarse en forma rutinaria en la práctica diaria ya que prolonga el tiempo del procedimiento endoscópico y su curva de aprendizaje es larga. 
Con los endoscopios de luz blanca de alta definición (E-HD) y la cromoendoscopia virtual (CE-virtual), se ha visto que la correlación es más alta. En un estudio de 234 pacientes, se encontró que la precisión diagnóstica, especificidad y sensibilidad fueron del $88 \%, 94 \%$ y $74,6 \%$ respectivamente. La sensibilidad de los E-HD es aún mayor cuando solo se consideran lesiones clínicamente significativas como la MIG incompleta y la displasia ${ }^{(56)}$.

Comparando los E-HD con CE-virtual mediante $\mathrm{NBI}$ (narrow-band imaging), en un estudio prospectivo multicéntrico realizado en cinco países occidentales, se mostró que el uso de NBI aumenta la precisión diagnóstica de la MIG en un $11 \%$ (NBI $94 \%$ Vs. E-HD 83\%) además, dicha tecnología aumentó significativamente la sensibilidad diagnóstica en este estudio del $55 \%$ al $87 \%$. El beneficio adicional de $\mathrm{NBI}$ en términos de precisión diagnóstica fue mayor en OLGIM III / IV que en OLGIM I / II (25\% frente a $15 \%$ respectivamente) ${ }^{(57)}$. En nuestro país, en un estudio realizado por Luna et al. donde se incluyeron 72 pacientes, se halló que los 5 pacientes con sospecha endoscópica de $\mathrm{MIG}$ con NBI, fueron todos confirmados luego por histología, mientras que ningún paciente sin sospecha demostró MIG en biopsias al azar ${ }^{(58)}$.

Una serie de otros estudios han demostrado que la CE-virtual es superior a los E-HD para detectar las CPCs, lo que llevó a algunos expertos a proponer un sistema de clasificación para determinar la extensión de la MIG sin tomar biopsias (Endoscopic Grading of Gastric Intestinal Metaplasia o EGGIM) cuyo aplicación en la práctica de rutina aun no está validada y requiere mayor investigación para ello ${ }^{(59)}$.

No obstante, la CE-virtual no está ampliamente disponible en nuestro país y la mayoría de los endoscopistas no están aún familiarizados con los patrones de imagen de la CE-virtual.

Xirouchakis et al., compararon la E-CLB con toma de biopsias según el protocolo de Sydney con la CE-virtual mediante NBI y toma de biopsias dirigidas. El 38\% de los pacientes tenían atrofia, de los cuales el 32,7\% fueron detectados con la E-CLB con toma de biopsias y $23,5 \%$ con NBI, el $21 \%$ tenía MIG de los cuales $16 \%$ fueron detectados con la E-CLB con toma de biopsias y $15.1 \%$ con NBI. Las precisiones diagnósticas de atrofia y MIG fueron del 93 y $90 \%$ para la E-CLB con toma de biopsias y 80 y $82 \%$ con NBI ${ }^{(60)}$. Estos resultados dejan en claro que la toma de biopsias según el protocolo de Sydney sigue siendo una excelente estrategia para detectar las CPCs.

En un estudio de casos y controles, Martínez et al. compararon la frecuencia de diagnóstico de atrofia cuando se utiliza el muestreo recomendado por el sistema actualizado de Sydney y la aplicación del sistema de OLGA con un muestreo sin tomar en cuenta dicho protocolo. Se encontró GA multifocal en el $42 \%$ de los pacientes versus el $26 \%$ del grupo control, lo cual significa un rendimiento del $61,8 \%$ más con el muestreo de 5 biopsias de Sydney ${ }^{(61)}$.

En su guía dominada MAPS II publicada el año pasado ${ }^{(35)}$, la Sociedad Europea de Endoscopia Gastrointestinal (ESGE), recomienda para una estadificación adecuada de las CPCs gástricas, una EDA diagnóstica por primera vez que debe incluir biopsias gástricas tanto para el diagnóstico de infección por H. pylori como para la identificación de etapas avanzadas de GA o MIG. Se deben tomar biopsias de al menos dos sitios topográficos: dos del antro (de la curvatura mayor y menor, a $3 \mathrm{~cm}$ del píloro) y dos del cuerpo (desde la curvatura menor, $4 \mathrm{~cm}$ proximal a la incisura, y desde la curvatura mayor, del medio). Las biopsias deben marcarse claramente en dos frascos separados. Se puede considerar agregar una biopsia de incisura para maximizar la detección de pacientes con afecciones precancerosas, especialmente en los casos en que la CE-virtual no está disponible para tomar biopsias dirigidas. También, se deben tomar biopsias adicionales de lesiones sospechosas visibles.

En resumen, los pacientes con CPCs como GA o MIG en estadios OLGA / OLGIM III / IV, tienen un riesgo significativamente mayor de progresar a CG por lo que estos pacientes se beneficiarían de un monitoreo endoscópico cercano y frecuente idealmente mediante E-HD para detectar LPCs en la etapa más temprana posible. Los pacientes con etapas de OLGA / OLGIM I / II, no se recomienda incluirlos en un programa de vigilancia endoscópica excepto si también presentan MIG incompleta y / o antecedentes familiares de CG.

\section{Vigilancia de las CPCs}

Los pacientes con MIG en un solo lugar pero con antecedentes familiares de CG o con MIG incompleta, la ESGE recomienda considerar la vigilancia endoscópica con CE-virtual y biopsias guiadas en 3 años. Los pacientes con etapas avanzadas de CPCc (cambios severos u OLGA / OLGIM III / IV) deben ser seguidos con una endoscopia de alta calidad cada 3 años. Los pacientes con etapas avanzadas de CPCs y con MI incompleta, $\mathrm{H}$. pylori persistente o, particularmente antecedentes familiares de CG pueden beneficiarse de un seguimiento más intensivo, cada 1 a 2 años ${ }^{(35)}$.

La Asociación Americana de Gastroenterología (AGA) en su última guía publicada en el mes de febrero de este año ${ }^{(62)}$, recomienda que los pacientes con signos de MIG de alto riesgo (MIG incompleta o MIG extensa) o cuando existen dudas sobre la calidad de la EDA inicial y / o quienes tienen un mayor riesgo general de CG (minorías raciales / étnicas, inmigrantes 
de regiones con alta incidencia de CG, o individuos con antecedentes familiares de primer grado de CG) pueden razonablemente repetir la EDA dentro de 1 año para la estratificación del riesgo. Los pacientes con MIG detectada accidentalmente, la AGA recomienda repetir la EDA en 3 a 5 años con visualización cuidadosa de la mucosa gástrica con toma de biopsias de cuerpo y antro y de cualquier lesión relevante.

\section{CONCLUSIONES}

Los pacientes con CPCs tienen un riesgo mayor que la población general de desarrollar CG, especialmente los que tienen etapas de OLGA / OLGIM III / IV o MIG incompleta y como efecto lógico, se debe ofrecer un seguimiento endoscópico a ellos.

Resulta importante aclarar que los estudios sobre la detección de CPCs y LPCs, fueron realizados por expertos, previa preparación de limpieza del estómago, y con un estudio meticuloso de la mucosa gástrica. En la práctica diaria, la mayoría de las EDAs se hacen sin una preparación previa, la cual demostró ser más eficaz ${ }^{(63,64)}$, sin eliminar adecuadamente todos los restos de la secreción mucosa pegada a la pared gástrica con el simple lavado y aspiración ${ }^{(65)}$, sin tomar el tiempo suficiente para evaluar de forma meticulosa y metodológica la mucosa gástrica, como la metodología de evaluación de la mucosa gástrica sugerida por Emura et al. (endoscopia sistemática alfanumérica codificada) ${ }^{(66)}$ y sin tomar siempre biopsias según el protocolo de Sydney en la primera EDA diagnóstica ${ }^{(67)}$. Estas limitaciones seguramente impactan negativamente en nuestra capacidad de reconocimiento de las CPCs y LPCs (cuya detección permite un seguimiento y manejo apropiado) y del CGT (cuyo tratamiento tiene un excelente pronóstico). Por lo tanto deberíamos ser más "científicos" a la hora de hacer las endoscopias y tomar biopsias no dirigidas según el protocolo de Sydney y biopsias dirigidas de lesiones visibles, de esa forma lograríamos reducir la tasa de mortalidad por el CG y ahorraríamos en hacer seguimiento endoscópico a pacientes sin riesgo de desarrollar CG y disminuiríamos la cantidad de endoscopias solicitadas por "control" o "second look" que por lo general tampoco se hacen tomando en cuenta estas cuestiones mencionadas.

Conflicto de intereses: Los autores declaran no tener ningún conflicto de intereses.

Financiamiento: Los autores declaran que no existió ningún tipo de financiamiento para el desarrollo de este trabajo.

\section{REFERENCIAS BIBLIOGRÁFICAS}

1. Bray F, Ferlay J, Soerjomataram I, Siegel RL, Torre LA, Jemal A. Global cancer statistics 2018: GLOBOCAN estimates of incidence and mortality worldwide for 36 cancers in 185 countries. CA Cancer J Clin. 2018;68(6):394-424.
2. Marqués-Lespier JM, González-Pons M, Cruz-Correa $M$. Current Perspectives on Gastric Cancer. Gastroenterol Clin North Am. 2016;45(3):413-28.

3. Petryszyn P, Chapelle N, Matysiak-Budnik T. Gastric cancer: Where are we heading? Dig Dis. 2020 Feb 17. doi: 10.1159/000506509.

4. Eusebi LH, Telese A, Marasco G, Bazzoli F, Zagari RM. Gastric cancer prevention strategies: A global perspective. J Gastroenterol Hepatol. 2020 Mar 17. doi: 10.1111/ jgh.15037.

5. Rodríguez-de-Santiago E, Hernanz N, Marcos-Prieto HM, de Jorge-Turrión MA, Barreiro-Alonso E, Rodríguez-Escaja $\mathrm{C}$, et al. A multicentric Spanish study on the characteristics and survival of gastric adenocarcinoma under the age of 60 . Gastroenterol Hepatol. 2019;42(10):595-603.

6. Sasako M. Progress in the treatment of gastric cancer in Japan over the last 50 years. Ann Gastroenterol Surg. 2020;4(1):2129. doi: 10.1002/ags3.12306. eCollection 2020 Jan.

7. Min YW, Min BH, Lee JH, Kim JJ. Endoscopic treatment for early gastric cancer. World J Gastroenterol. 2014;20(16):456673. doi: 10.3748/wjg.v20.i16.4566.

8. Oliveira FJ, Ferrão H, Furtado E, Batista H, Conceição L. Early gastric cancer: Report of 58 cases. Gastric Cancer. 1998;1(1):51-56.

9. Kurtz RC, Sherlock P. The diagnosis of gastric cancer. Semin Oncol. 1985;12(1):11-8.

10. Lauren P. The two histological main types of gastric carcinoma: diffuse and so-called intestinal-type carcinoma. An attempt at a histo-clinical classification. Acta Pathol Microbiol Scand. 1965;64:31-49.

11. Correa P. Gastric cancer: overview. Gastroenterol Clin North Am. 2013;42(2):211-7. doi: 10.1016/j.gtc.2013.01.002.

12. Gisbert JP. Helicobacter pylori-related diseases. Gastroenterol Hepatol. 2016;39 Suppl 1:36-46. doi: 10.1016/S02105705(16)30173-X.

13. Seoane A, Bessa X, Balleste B, O'Callaghan E, Panadès A, Alameda $F$, et al. Helicobacter pylori and gastric cancer: relationship with histological subtype and tumor location. Gastroenterol Hepatol. 2005;28(2):60-4.

14. Neumann WL, Coss E, Rugge M, Genta RM. Autoimmune atrophic gastritis--pathogenesis, pathology and management. Nat Rev Gastroenterol Hepatol. 2013;10:529-541.

15. Zullo A, Hassan C, Romiti A, Giusto M, Guerriero C, Lorenzetti $\mathrm{R}$, et al. Follow-up of intestinal metaplasia in the stomach: When, how and why. World J Gastrointest Oncol. 2012;4(3):30-6. doi: 10.4251/wjgo.v4.i3.30.

16. Sobala GM1, O'Connor HJ, Dewar EP, King RF, Axon AT, Dixon MF. Bile reflux and intestinal metaplasia in gastric mucosa. J Clin Pathol. 1993;46(3):235-40.

17. Huang RJ, Choi AY, Truong CD, Yeh MM, Hwang JH. Diagnosis and Management of Gastric Intestinal Metaplasia: Current Status and Future Directions. Gut Liver. 2019;13(6):596-603. doi: 10.5009/gnl19181.

18. González CA, Sanz-Anquela JM, Companioni O, Bonet C, Berdasco M, López $C$, et al. Incomplete type of intestinal metaplasia has the highest risk to progress to gastric cancer: results of the Spanish follow-up multicenter study. J Gastroenterol Hepatol. 2016;31(5):953-8. doi: 10.1111/ jgh.13249.

19. Shao L, Li P, Ye J, Chen J, Han Y, Cai J, et al. Risk of gastric cancer among patients with gastric intestinal metaplasia. Int J Cancer. 2018;143(7):1671-1677. doi: 10.1002/ijc.31571. Epub 2018 Jul 26.

20. Spence AD, Cardwell CR, McMenamin ÚC, Hicks BM, Johnston BT, Murray LJ, et al. Adenocarcinoma risk in gastric atrophy and intestinal metaplasia: a systematic review. BMC Gastroenterol. 2017;17(1):157. doi: 10.1186/s12876-0170708-4.

21. Zhang L, Liu Y, You P, Feng G. Occurrence of gastric cancer in patients with atrophic gastritis during long-term followup. Scand J Gastroenterol. 2018;53(7):843-848. doi: 10.1080/00365521.2018.1477987.

22. Kaji K1, Hashiba A, Uotani C, Yamaguchi Y, Ueno T, Ohno K, et al. Grading of Atrophic Gastritis is Useful for Risk 
Stratification in Endoscopic Screening for Gastric Cancer. Am J Gastroenterol. 2019;114(1):71-79. doi: 10.1038/s41395018-0259-5.

23. Rugge M, Genta RM. Staging and grading of chronic gastritis. Hum Pathol. 2005;36(3):228-33.

24. Cassaro M, Rugge M, Gutierrez O, Leandro G, Graham DY, Genta RM. Topographic patterns of intestinal metaplasia and gastric cancer. Am J Gastroenterol. 2000;95:1431-1438.

25. Sakitani K, Hirata Y, Watabe H, Yamada A, Sugimoto $\mathrm{T}$, Yamaji $\mathrm{Y}$, et al. Gastric cancer risk according to the distribution of intestinal metaplasia and neutrophil infiltration. J Gastroenterol Hepatol. 2011;26(10):1570-5. doi: 10.1111/j.1440-1746.2011.06767.x.

26. Corti RE. Gastritis. Acta Gastroenterol Latinoam. 2001;4:265-275.

27. Corti RE, Cohen H. Temas escogidos en patologia gastroduodenal. (Cap 8) Buenos Aires: Ed Content Medicine; 2012.

28. Gómez E, Mella JM, Pereyra L, González R, Medrano MA, Fischer $\mathrm{C}$, et al. Riesgo de lesiones gástricas entre pacientes caucásicos y asiáticos con epigastralgia en un hospital privado de la comunidad en Argentina. Acta Gastroenterol Latinoam. 2010;40 Sup 1:S44.

29. Song H, Ekheden IG, Ploner A, Ericsson J, Nyren O, Ye W. Family history of gastric mucosal abnormality and the risk of gastric cancer: a population-based observational study. Int J Epidemiol. 2018;47(2):440-449. doi: 10.1093/ije/dyx238.

30. de Vries AC, van Grieken NC, Looman CW, Casparie MK, de Vries E, Meijer GA, et al. Gastric cancer risk in patients with premalignant gastric lesions: a nationwide cohort study in the Netherlands. Gastroenterology. 2008;134(4):945-52. doi: 10.1053/j.gastro.2008.01.071.

31. Song H, Ekheden IG, Zheng Z, Ericsson J, Nyrén O, Ye W. Incidence of gastric cancer among patients with gastric precancerous lesions: observational cohort study in a low risk Western population. BMJ. 2015;351:h3867. doi: 10.1136/ bmj.h3867.

32. Li D, Bautista MC, Jiang SF, Daryani P, Brackett M, Armstrong MA, et al. Risks and Predictors of Gastric Adenocarcinoma in Patients with Gastric Intestinal Metaplasia and Dysplasia: A Population-Based Study. Am J Gastroenterol. 2016;111(8):1104-13. doi: 10.1038/ajg.2016.188.

33. Shichijo $S$, Hirata $Y$, Sakitani $K$, Yamamoto $S$, Serizawa $T$, Niikura R, et al. Distribution of intestinal metaplasia as a predictor of gastric cancer development. J Gastroenterol Hepatol. 2015;30(8):1260-4. doi: 10.1111/jgh.12946.

34. Akbari M, Tabrizi R, Kardeh S, Lankarani KB. Gastric cancer in patients with gastric atrophy and intestinal metaplasia: A systematic review and meta-analysis. PLoS One. 2019;14(7):e0219865. doi: 10.1371/journal.pone.0219865.

35. Pimentel-Nunes $P$, Libânio D, Marcos-Pinto R, Areia M, Leja $M$, Esposito G, et al. Management of epithelial precancerous conditions and lesions in the stomach (MAPS II): European Society of Gastrointestinal Endoscopy (ESGE), European Helicobacter and Microbiota Study Group (EHMSG), European Society of Pathology (ESP), and Sociedade Portuguesa de Endoscopia Digestiva (SPED) guideline update 2019. Endoscopy. 2019;51(4):365-388. doi: 10.1055/a0859-1883.

36. Quach DT, Hiyama T, Gotoda T. Identifying high-risk individuals for gastric cancer surveillance from western and eastern perspectives: Lessons to learn and possibility to develop an integrated approach for daily practice. World J Gastroenterol. 2019;25(27):3546-3562. doi: 10.3748/wjg. v25.i27.3546.

37. Duits LC, van der Wel MJ, Cotton CC, Phoa KN, Ten Kate FJW, Seldenrijk CA, et al. Patients With Barrett's Esophagus and Confirmed Persistent Low-Grade Dysplasia Are at Increased Risk for Progression to Neoplasia. Gastroenterology. 2017;152(5):993-1001.e1. doi: 10.1053/j. gastro.2016.12.008.

38. Zhao G, Xue M, Hu Y, Lai S, Chen S, Wang L, et al. How commonly is the diagnosis of gastric low grade dysplasia upgraded following endoscopic resection? A meta-analysis PLoS One. 2015;10:e0132699.
39. Luna P, Pereyra L, Amante MF, Casas G, Mohaidle A, Gómez EJ, et al. Determinadas características endoscópicas y clínicas son capaces de predecir la presencia de displasia en pacientes con metaplasia intestinal gástrica evidente a la visión endoscópica. Acta Gastroenterol Latinoam. 2013;43 Sup 2:S47.

40. Rugge M, Meggio A, Pennelli G, Piscioli F, Giacomelli L, De Pretis G, et al. Gastritis staging in clinical practice: the OLGA staging system. Gut. 2007;56(5):631-6.

41. Crafa P, Russo M, Miraglia C, Barchi A, Moccia F, Nouvenne A, et al. From Sidney to OLGA: an overview of atrophic gastritis. Acta Biomed. 2018;89(8-S):93-99. doi: 10.23750/ abm.v89i8-S.7946.

42. Rugge $M$, de Boni $M$, Pennelli $G$, de Bona $M$, Giacomelli $L$, Fassan $M$, et al. Gastritis OLGA-staging and gastric cancer risk: a twelve-year clinico-pathological follow-up study. Aliment Pharmacol Ther. 2010;31(10):1104-11. doi: 10.1111/j.1365-2036.2010.04277.x.

43. Capelle LG, de Vries AC, Haringsma J, Borg FT, de Vries RA, Bruno MJ, et al. The staging of gastritis with the OLGA system by using intestinal metaplasia as an accurate alternative for atrophic gastritis. Gastrointest Endosc. 2010;71(7):1150-8. doi: 10.1016/j.gie.2009.12.029.

44. Capelle LG, Haringsma J, de Vries AC, Steyerberg EW, Biermann $\mathrm{K}$, van Dekken $\mathrm{H}$, et al. Narrow band imaging for the detection of gastric intestinal metaplasia and dysplasia during surveillance endoscopy. Dig Dis Sci. 2010;55(12):3442-8. doi: 10.1007/s10620-010-1189-2.

45. Uemura $N$, Okamoto $S$, Yamamoto $S$, Matsumura $N$ Yamaguchi S, Yamakido $M$, et al. Helicobacter pylori infection and the development of gastric cancer. $N$ Engl J Med. 2001;345(11):784-9.

46. Yun CY, Kim N, Lee J, Lee JY, Hwang YJ, Lee HS, et al. Usefulness of OLGA and OLGIM system not only for intestinal type but also for diffuse type of gastric cancer, and no interaction among the gastric cancer risk factors. Helicobacter. 2018;23(6):e12542. doi: 10.1111/hel.12542.

47. Shin SY, Kim JH, Chun J, Yoon YH, Park H. Chronic atrophic gastritis and intestinal metaplasia surrounding diffuse-type gastric cancer: Are they just bystanders in the process of carcinogenesis? PLoS One. 2019;14(12):e0226427. doi: 10.1371/journal.pone.0226427.

48. Kujaruk M, Cabanne A, Doweck J, Filippa L, Bestani C, Dulcich $F$, et al. Estado histopatologico de la mucosa gástrica en estomagos resecados por adenocarcinoma. Acta Gastroenterol Latinoam. 2016;46 Sup 1:S7.

49. Chen WF, Li QL, Zhou PH, Xu MD, Zhang YQ, Zhong YS, et al. Clinical value of different magnifying chromoendoscopy methods in screening gastric precancerous lesions and early cancers. Zhonghua Wei Chang Wai Ke Za Zhi. 2012;15(7):662-7.

50. Pittayanon R, Rerknimitr R. Role of digital chromoendoscopy and confocal laser endomicroscopy for gastric intestinal metaplasia and cancer surveillance. World J Gastrointest Endosc. 2012;4(10):472-8. doi: 10.4253/wjge.v4.i10.472.

51. Lim LG, Yeoh KG, Srivastava S, Chan YH, The M, Ho KY. Comparison of probe-based confocal endomicroscopy with virtual chromoendoscopy and white-light endoscopy for diagnosis of gastric intestinal metaplasia. Surg Endosc. 2013;27(12):4649-55. doi: 10.1007/s00464-013-3098-x.

52. Areia M, Amaro P, Dinis-Ribeiro M, Cipriano MA, Marinho C, Costa-Pereira A, et al. External validation of a classification for methylene blue magnification chromoendoscopy in premalignant gastric lesions. Gastrointest Endosc. 2008;67:1011-1018.

53. Calle Astudillo G, Jerves T, Pesántez L, Calle P, Gutiérrez Á, Calle G. Utilidad de las biopsias gástricas rutinarias y de la tinción con azul de metileno en el diagnóstico de la metaplasia intestinal en mayores de 40 años. Acta Gastroenterol Latinoam. 2013;43(3):189-97.

54. Song KH, Hwang JA, Kim SM, Ko HS, Kang MK, Ryu KH, et al. Acetic acid chromoendoscopy for determining the extent of gastric intestinal metaplasia. Gastrointest Endosc. 2017;85(2):349-356. doi: 10.1016/j.gie.2016.07.064. 
55. Yep-Gamarra V, Díaz-Vélez C, Araujo I, Ginès À, FernándezEsparrach G. Usefulness of systematic chromoendoscopy with a double dye staining technique for the detection of dysplasia in patients with premalignant gastric lesions. Gastroenterol Hepatol. 2016;39(2):49-54. doi: 10.1016/j. gastrohep.2015.08.005.

56. Panteris V, Nikolopoulou S, Lountou A, Triantafillidis JK. Diagnostic capabilities of high-definition white light endoscopy for the diagnosis of gastric intestinal metaplasia and correlation with histologic and clinical data. Eur J Gastroenterol Hepatol. 2014;26(6):594-601. doi: 10.1097/ MEG.0000000000000097.

57. Pimentel-Nunes P, Libanio D, Lage J, Abrantes D, Coimbra $M$, Esposito $G$, et al. A multicenter prospective study of the real-time use of narrow-band imaging in the diagnosis of premalignant gastric conditions and lesions. Endoscopy. 2016;48:723-730.

58. Luna P, Pereyra L, Casas G, Mella JM, Mohaidle A, Gonzalez $R$, et al. Es posible identificar la mucosa gástrica normal e infectada por helicobacter pylori, así como la metaplasia intestinal gástrica utilizando endoscopía con NBI (narrow band imaging o imagen de banda estrecha) sin magnificación? un estudio prospectivo. Acta Gastroenterol Latinoam. 2012;42 Sup 2:S52.

59. Marcos P, Brito-Gonçalves G, Libânio D, Pita I, Castro R, Sá I, et al. Endoscopic grading of gastric intestinal metaplasia on risk assessment for early gastric neoplasia: can we replace histology assessment also in the West? Gut. 2020 Feb 12. doi: 10.1136/gutjinl-2019-320091.

60. Xirouchakis E, Laoudi F, Tsartsali L, Spiliadi C, Georgopoulos SD. Screening for gastric premalignant lesions with narrow band imaging, white light and updated Sydney protocol or both? Dig Dis Sci. 2013;58(4):1084-90. doi: 10.1007/ s10620-012-2431-x.

61. Martinez D, William O, Orlando R. Impacto del sistema OLGA en la detección de gastritis crónica atrófica en Colombia: un estudio de casos y controles. Rev Col Gastroenterol. 2016;31(4):360-367.
62. Gupta S, Li D, El Serag HB, Davitkov P, Altayar O, Sultan S, et al. AGA Clinical Practice Guidelines on Management of Gastric Intestinal Metaplasia. Gastroenterology. 2020;158(3):693702. doi: 10.1053/j.gastro.2019.12.003.

63. Elvas L, Areia M, Brito D, Alves S, Saraiva S, Cadime AT. Premedication with simethicone and $\mathrm{N}$-acetylcysteine in improving visibility during upper endoscopy: a double-blind randomized trial. Endoscopy. 2017;49(2):139-145. doi: 10.1055/s-0042-119034.

64. Mahawongkajit P, Kanlerd A. A prospective randomized controlled trial comparing simethicone, $\mathrm{N}$-acetylcysteine, sodium bicarbonate and peppermint for visualization in upper gastrointestinal endoscopy. Surg Endosc. 2020 Feb 3. doi: 10.1007/s00464-020-07397-8.

65. Córdova H, Sánchez-Montes C, Delgado-Guillena PG Morales VJ, Sendino O, González-Suárez B, et al. Quality indicators for esophagogastroduodenoscopy: A comparative study of outcomes after an improvement programme in a tertiary hospital. Gastroenterol Hepatol. 2017;40(9):587594. doi: 10.1016/j.gastrohep.2017.05.007.

66. Emura F, Sharma P, Arantes V, Cerisoli C, Parra-Blanco A, Sumiyama K, et al. Principles and practice to facilitate complete photodocumentation of the upper gastrointestinal tract: World Endoscopy Organization position statement. Dig Endosc. 2020;32(2):168-179. doi: 10.1111/den.13530.

67. Crafa P, Russo M, Miraglia C, Barchi A, Moccia F, Nouvenne A, et al. From Sidney to OLGA: an overview of atrophic gastritis. Acta Biomed. 2018;89(8-S):93-99. doi: 10.23750/ abm.v89i8-S.7946.

\section{Correspondencia:}

Muhannad Sarem

Unidad de Endoscopia. Hospital Dr. Diego Thompson. San Martín.

Provincia de Buenos Aires. Argentina.

E-mail:msarem@hotmail.com 